\title{
Thermal Imaging of Uranus: Upper-Tropospheric Temperatures One Season After Voyager
}

\author{
Glenn S. Orton ${ }^{1}$ \\ Leigh N. Fletcher ${ }^{2}$ \\ Therese Encrenaz ${ }^{3}$ \\ Cedric Leyrat ${ }^{3}$ \\ Henry G. Roe ${ }^{4}$ \\ Takuya Fujiyoshi ${ }^{5}$ \\ Eric Pantin 6
}

${ }^{1}$ MS 183-501, Jet Propulsion Laboratory, California Institute of Technology, 4800 Oak Grove Drive, Pasadena, California 91109, USA

${ }^{2}$ Atmospheric, Oceanic \& Planetary Physics, Department of Physics, University of Oxford, Clarendon Laboratory, Parks Road, Oxford OX1 3PU, United Kingdom ${ }^{3}$ LEISA, Observatoire de Paris, F-92195, Meudon, France ${ }^{4}$ Lowell Observatory, 1400 West Mars Hill Road, Flagstaff, Arizona 86001, USA. ${ }^{5}$ Subaru Telescope, National Astronomical Observatory of Japan, National Institutes of Natural Sciences, 650 N. A'ohoku Place, Hilo, Hawaii 96720, USA ${ }^{6}$ Centre d'Études Atomiques, Saclay, 91109 Gif-sur-Yvette, France

\begin{abstract}
We report on 18-25 $\mu \mathrm{m}$ thermal imaging of Uranus that took place between 2003 and 2011, a time span roughly one season after the thermal maps made by the Voyager-2 IRIS experiment in 1986. We re-derived meridional variations of temperature and para $-\mathrm{H}_{2}$ fraction from the Voyager experiment and compared these with the thermal images, which are sensitive to temperatures in the upper troposphere of Uranus around the 70-400 mbar atmospheric pressure range. The thermal images display a maximum of $3 \mathrm{~K}$ of equivalent temperature changes across the disk, and they are consistent with the temperature distribution measured by the Voyager IRIS experiment. This implies that there has been no detectable change of the meridional distribution of upper-tropospheric / lower-stratospheric temperatures over a season. This is inconsistent with seasonally dependent radiative-convective-dynamical models and full global climate models that predict some variability with season if the effective temperature is meridionally constant. We posit that the effective temperature of Uranus could be meridionally variable, with the additional possibility that even the small temperature variations predicted by the GCMs are overestimated.
\end{abstract}




\section{Introduction}

With its $98^{\circ}$ rotational axis tilt from its orbital plane, the atmosphere of Uranus is subject to extremes of seasonal variability in 84-year cycles of daylight and darkness. The thermal structure and response of the atmosphere to these extremes were determined only once in 1986 with close-up thermal spectroscopy of Uranus by the Voyager-2 IRIS experiment (e.g. Flasar et al. 1987, Conrath et al. 1990). It measured the north pole near winter solstice (planetocentric solar longitude $\mathrm{L}_{\mathrm{s}}=273^{\circ}$ ) in the midst of its long winter darkness, yielding upper tropospheric (80mbar) temperatures that were 1-2 $\mathrm{K}$ warmer than at the equator (Conrath et al. 1998). In order to determine the effect of extremes of variable sunlight on different latitudes in Uranus, we needed to determine temperatures near the same uppertroposphere levels as measured by the IRIS experiment. This is not possible from Earth using the same 30-50 $\mu \mathrm{m}$ wavelength range as the Voyager IRIS experiment because of strong absorption by water vapor in our own atmosphere. However, we can measure temperatures in a portion of the 75-650 mbar pressure range covered by the Voyager IRIS experiment from the $18-25 \mu \mathrm{m}$ spectral range that is accessible from ground-based observatories. The IRIS experiment was also sensitive to the relative abundances of para $-\mathrm{H}_{2}$ vs. ortho $-\mathrm{H}_{2}$ ratio, parameterized by the para $-\mathrm{H}_{2}$ fraction, whose departures from local equilibrium can serve as a tracer of vertical motion. Ground-based observations that extend to $25 \mu \mathrm{m}$ are also sensitive to the para $-\mathrm{H}_{2}$ fraction at one level, around 200 mbar of atmospheric pressure. We report here Earth-based spatially resolved thermal images of Uranus between 18 and 25 $\mu \mathrm{m}$ taken between 2003 and $2011\left(\mathrm{~L}_{\mathrm{s}}=354-14^{\circ}\right)$. Following the Voyager IRIS measurements by 17-25 years, they map upper-tropospheric temperatures and the para $-\mathrm{H}_{2}$ fraction at a significantly different season than Voyager, near the northern spring equinox $\left(\mathrm{L}_{s}=0^{\circ}\right)$.

\section{Observations}

We assume that molecular hydrogen $\left(\mathrm{H}_{2}\right)$ is well mixed in the atmosphere of Uranus, because the spectral region we sample is insensitive to the condensation level of $\mathrm{CH}_{4}$ whose unsaturated mixing ratio in the deeper atmosphere could be as high as $4 \%$ (Lindal et al. 1987, Rages et al. 1991, Sromovsky et al. 2011). Thus, variability in observed radiances from regions dominated by the opacity of $\mathrm{H}_{2}$ can be used to determine the variability of temperatures. Figure 1 illustrates that the 17-25 $\mu \mathrm{m}$ spectrum of Uranus is dominated by the collision-induced opacity of $\mathrm{H}_{2}$. Figure 2 displays contribution functions that illustrate that this spectral range is sensitive to the vertical temperature structure in the pressure range of 70 to $400 \mathrm{mbar}$. Furthermore, it is also sensitive to the para- $\mathrm{H}_{2}$ fraction because the collisioninduced absorption in this region is composed of contributions of the broad rotational features arising from the $\mathrm{S}(0)\left(\right.$ para $\left.-\mathrm{H}_{2}\right)$ and $\mathrm{S}(1)\left(\right.$ ortho $\left.-\mathrm{H}_{2}\right)$ transitions. Thus, an intercomparison of radiances from these wavelengths can determine the para $-\mathrm{H}_{2}$ fraction and its variability at 50-300 mbar pressures over different latitudes in Uranus. Although there are intervals in the 9-11 $\mu \mathrm{m}$ region that may well be dominated by very weak $\mathrm{H}_{2}$ collision-induced absorption and are thus sensitive to 
temperatures as deep as the 2-bar pressure level (see Orton et al. 2014b), radiances in that region are much weaker and their detection from ground-based facilities is impractical.

Images of Uranus were obtained with filters associated with the mid-infrared facility instruments shown in Table 1, which also summarizes the details of each observing run. Figure 3 displays the final image products. All images were made with standard chopping and nodding in order to subtract background sky emission on time scales of seconds and minutes, respectively. All the images were made in association with other targets for each night, and the required integration and associated overhead times made it impossible to observe the planet using more than one filter per night. The sequence of the observations was driven by a combination of the overhead water-vapor burden (a low value permitted longer-wavelength observations) and what had already been observed, starting with what we judged to be the easiest detections.

Table 1. Summary of Uranus observations.

\begin{tabular}{|c|c|c|c|}
\hline $\begin{array}{c}\text { Central Wavelength } \\
(\text { FWHM of filter })\end{array}$ & Source & UT Date & $\begin{array}{c}\text { Start - End Times } \\
\text { (UTC) }\end{array}$ \\
\hline $18.7 \mu \mathrm{m}(0.83 \mu \mathrm{m})$ & VLT/VISIR & 2006 Sep. 2 & $4: 29-7: 21$ \\
\hline $18.7 \mu \mathrm{m}(0.83 \mu \mathrm{m})$ & VLT/VISIR & 2006 Sep. 3 & $4: 03-6: 35$ \\
\hline $19.5 \mu \mathrm{m}(0.35 \mu \mathrm{m})$ & VLT/VISIR & 2009 Aug. 27 & $5: 48-10: 06$ \\
\hline $20.5 \mu \mathrm{m}(0.97 \mu \mathrm{m})$ & Subaru/COMICS & 2011 Aug. 27 & $11: 34-11: 54$ \\
\hline $22.0 \mu \mathrm{m}(1.60 \mu \mathrm{m})$ & Keck/LWS & 2003 Jul. 20 & $13: 49-14: 53$ \\
\hline $24.5 \mu \mathrm{m}(0.16 \mu \mathrm{m})$ & Subaru/COMICS & 2008 Sep. 15 & $9: 16-9: 51$ \\
\hline $24.5 \mu \mathrm{m}(0.16 \mu \mathrm{m})$ & Subaru/COMICS & 2008 Sep. 16 & $8: 27-9: 31$ \\
\hline
\end{tabular}

$18.7 \mu \mathrm{m}$ : Some of our initial thermal images of Uranus were made with an $18.7-\mu \mathrm{m}$ filter during the second half of two nights on the Very Large Telescope (VLT) UT3, using the VISIR instrument (Lagage et al 2004). Some images on 2006 Sep. 2 were acquired after 7:21 UT but ultimately discarded because of degraded angular resolution. All VISIR images were acquired with the plate scale set at 0.075 arcsec per pixel. The image for this date shown in Fig. 3 is the result of four co-added observations, each taking 30 minutes of real time. Similarly, the 18.7- $\mu$ m image shown in Fig. 3 for 2006 Sep. 3 is the result of coadding another set of four individual images with the same integration time. Coadding these images that were acquired over a time span of 2.5 - 3.0 hours means that longitudinal (zonal) variability is smeared over $\sim 50-60^{\circ}$ of longitude, assuming a 17.24-hour rotational period, as adopted by the International Astronomical Union's Working Group on Cartographic Coordinates and Rotational Elements (Archinal et al. 2011). No variability in longitude is evident above the noise of any of these individual images. The mean signal-to-noise ratio (SNR) per pixel in the coadded image for Sep. 2 shown in Fig. 3 is 3.9; the mean SNR per pixel in the corresponding image for Sep. 3 is 3.8. Comparison observations were made with standard star tau Aquarii. 
$19.5 \mu \mathrm{m}$ : We acquired additional images at $19.5 \mu \mathrm{m}$ at the VLT's UT3 facility with VISIR in queue time in 2009. The 19.5- $\mu \mathrm{m}$ image shown in Fig. 3 is the product of coadding eight individual frames, each of which represented integrations taking 20 minutes of real time. The mean SNR per pixel in the coadded image shown in Fig. 3 is 3.5. Unfortunately, no standard-star observations were acquired on this run.

$20.5 \mu \mathrm{m}$ : We imaged Uranus at $20.5 \mu \mathrm{m}$ at the Subaru Telescope using the COMICS instrument (Kataza et al. 2000) on an extremely dry night. The COMICS plate scale is fixed at 0.13 arcsec per pixel. We collected a set of four images, each of which took 5 minutes of real time. The integration times were limited by the random-access memory (RAM) size. The mean SNR per pixel in the coadded images shown in Fig. 3 is 2.9. Comparison observations were made of standard star alpha Ceti.

$22.0 \mu \mathrm{m}$ : We imaged Uranus at $22.0 \mu \mathrm{m}$ at the Keck-1 Telescope using the LongWavelength Spectrometer (LWS), which was described in an earlier form by Jones and Puetter (1993). The observations were acquired with individual 10-msec exposures; a total of 18 minutes of exposure was acquired on Uranus and coadded, with an equal amount of time taking comparison measurements of the nearby sky. The mean SNR per pixel in ten coadded image shown in Fig. 3 is $\sim 6$. The comparison star was tau Aquarii. The observations of Uranus and tau Aquarii were made during variable precipitable water vapor conditions and were completed just before fog obscured the sky.

$24.5 \mu \mathrm{m}$ : Images were obtained at the Subaru Telescope using the $24.5-\mu \mathrm{m}$ filter of the COMICS instrument (the only instrument at a $\geq 8$-meter-class telescope with this capability). We collected a set of seven images on Sep. 15 and eleven images on Sep. 16 , each of which took 5 minutes of real time, just as for the 20.5- $\mu$ m images. The mean SNR per pixel in the coadded images shown in Fig. 3 is 4.6 for Sep. 15 and 3.4 for Sep. 16. Comparison observations were made of standard star beta Geminorum on Sep. 15 and gamma Aquillae on Sep. 16.

We note that we attempted similar observations of Uranus on other dates, but the optical path was not sufficiently dry to allow what we considered a bare minimum SNR per pixel $(>2)$ to allow useful analysis. We also did not attempt to image Uranus in filters whose central wavelengths were between 17 and $18 \mu \mathrm{m}$, as we have for our studies of thermal imaging of Jupiter and Saturn (see Fletcher et al. 2009, for example). Although this region senses temperatures at somewhat lower pressures than at the longer wavelengths, we would expect very low radiances from the planet and would have been much less likely to acquire any images meeting our SNR criterion.

Our initial intent was to use the standard-star observations to provide a radiometric standard for the observations of Uranus, using the template models of Cohen et al. (1999) and references therein. However the 2006 observations at $18.7 \mu \mathrm{m}$ followed a period of unusual torrential rain, after the amount of water vapor in the optical path was varying rapidly, as noted by Orton et al. (2007a) for the observations of 
Neptune taken on the same nights. Furthermore, there was no measurement of a standard star made with the $19.5-\mu \mathrm{m}$ images of Uranus by the VLT staff in queue time, and the standard star associated with the $20.5-\mu \mathrm{m}$ image at Subaru was made at a position that was 0.4 airmasses different from the Uranus observations. Given these difficulties and the fact that we are primarily comparing changes between the date of these observations and the results of observations by the Voyager-2 IRIS experiment, as explained in Section 3, we adopted an approach that normalized the total flux to the expected output from a model based on a simulation of the global mean radiance generated by the distribution of temperatures and para- $\mathrm{H}_{2}$ derived by the Voyager IRIS experiment (Conrath et al. 1998), as described in section 3.1. Because our focus was on making this comparison we did not attempt to produce deconvolved images using the point-spread functions implied by the stellar images. This enabled us to preserve a record of the observations without introducing artifacts that would be related to the deconvolution.

\section{Analysis}

\subsection{Revised analysis of Voyager IRIS data}

Because the images were taken so far apart in time and covered only five discrete wavelengths, we refrained from inverting the data to derive upper-tropospheric temperatures and a para $-\mathrm{H}_{2}$ fraction at one level in favor of a more direct comparison with the Voyager-2 IRIS results to search for evidence of seasonal or other changes in time since 1986. Furthermore, there have been updates to models of $\mathrm{H}_{2}$ collision-induced absorption (CIA), which controls the long-wavelength thermal spectrum, since the analysis of IRIS data by Conrath et al. (1998), so we rederived the zonal-mean temperature and para $-\mathrm{H}_{2}$ fraction profiles from the same data. We used the tables created by Orton et al. (2007b) for $\mathrm{H}_{2}-\mathrm{H}_{2}$ CIA and the equations of Borysow et al. (1988) for $\mathrm{H}_{2}$ - $\mathrm{He}$ CIA and Borysow et al. (1986) for $\mathrm{H}_{2}$ $\mathrm{CH}_{4} \mathrm{CIA}$. In addition, we use $a b$ initio calculations of CIA absorption coefficients for a range of para $-\mathrm{H}_{2}\left(\mathrm{f}_{\mathrm{p}}\right)$ fractions appropriate to the ice giants, which were sampled on a $\Delta \mathrm{f}_{\mathrm{p}}$ grid of 0.02 (M. Gustafsson, personal communication). We assumed a ratio of $85 \% \mathrm{H}_{2}$ to $15 \%$ He by number, following the joint analysis of Voyager-2 IRIS and radio-occultation observations by Conrath et al. (1987). Methane is added to this mixture with a volume mixing ratio of $3.2 \%$ in the deep atmosphere, consistent with the global-mean results of Karkoschka and Tomasko $(2009,2011)$, and obeying saturation equilibrium vapor pressures above the condensation level and matching the Spitzer-derived stratospheric abundance of Orton et al. (2014a), corresponding to a volume-mixing ratio of $1.6 \times 10^{-5}$ in the lower stratosphere. No significant changes to our quantitative results arose from variations of the deep atmospheric $\mathrm{CH}_{4}$ mixing ratio or assuming less than $100 \%$ humidity for $\mathrm{CH}_{4}$ above the condensation level.

The Voyager-2/IRIS data were extracted from the VG2001 archive on the Planetary Data System (http://pds-rings.seti.org/vol/VG_2001/URANUS/). The dataset 
extends over the four days surrounding closest approach (1986 January 22-26), corresponding to distances ranging from 112 Uranus radii $\left(\mathrm{R}_{U}\right)$ inbound to $4.2 \mathrm{R}_{U}$ at closest approach on January 24 to $106 \mathrm{R}_{U}$ outbound. The data were generally grouped into hemispheric maps, one of the southern summer hemisphere (subspacecraft latitude near $57^{\circ} \mathrm{S}$ during the inbound leg) and one of the northern winter hemisphere (sub-spacecraft latitude near $68^{\circ} \mathrm{N}$ during the outbound leg). The data zenith angles (along with the distance to the object) are shown in Fig. 4. Following Conrath et al. (1998), we use these data in their entirety, binning spectra in $10^{\circ}$ wide latitude bins with a $5^{\circ}$ latitude step, retaining only zenith angles within $\pm 15^{\circ}$ of the mean zenith angle in a particular bin, and only those spectra with a 'zero' reject code (signifying that the interferogram was good). Only the $200-400 \mathrm{~cm}^{-1}$ spectral range was retained, sampled on a $2.15-\mathrm{cm}^{-1}$ grid with a spectral resolution of $4.3 \mathrm{~cm}^{-}$ 1 . Figure 5 illustrates examples of the warmest and coolest spectra.

The zonally (longitudinally) averaged IRIS spectra were inverted using an optimal estimation retrieval algorithm, NEMESIS (Irwin et al., 2008), deriving continuous profiles of atmospheric temperature and para-hydrogen fraction. The sources of line data appropriate to the ice giants, and their conversion to k-distributions (ranking absorption coefficients according to their frequency distributions within a defined interval) to maximize the efficiency of the forward model, were described in detail by Fletcher et al. (2014). The a priori assumptions (the "prior") consisted of the vertical temperatures and molecular abundances presented by Orton et al. $(2014 \mathrm{a}, \mathrm{b})$, and the para $-\mathrm{H}_{2}$ fraction calculated at thermochemical equilibrium. Multiple retrievals were performed to test the sensitivity of the retrieved state to the prior, selecting smoothing constraints that provided physically plausible vertical profiles whilst also fitting the data to within the uncertainty. Aerosol opacity was assumed to be negligible at these far-infrared wavelengths. Fig. 6 shows the zonally-averaged temperature structure, $\mathrm{T}(\mathrm{p})$, and the associated lapse rate $\mathrm{dT} / \mathrm{dz}$, plus the para- $\mathrm{H}_{2}$ fraction $\left(\mathrm{f}_{\mathrm{p}}\right)$ and its deviation from thermal equilibrium $\left(\mathrm{f}_{\mathrm{p}, \mathrm{eqm}}\right)$. Typical tropopause-level uncertainties from the spectral inversions were $<1 \mathrm{~K}$ for temperature and $<0.02$ for the para- $\mathrm{H}_{2}$ fraction.

The zonal mean temperatures compare favorably with those of Conrath et al., (1998), with two broad minima at $30-40^{\circ} \mathrm{N}$ and $20-50^{\circ} \mathrm{S}$, with the coolest temperatures (50-51 $\mathrm{K}$ at the tropopause) observed at northern mid-latitudes. Indeed, the tropopause appears to be highest at $40^{\circ} \mathrm{N}$. A very slight asymmetry is observed between the temperatures of the two poles, with a stronger lapse rate in the lower stratosphere over the summer pole than the winter pole, consistent with the increased insolation heating the mid-stratosphere. At the tropopause (100-140 mbar, Fig. 6a) however, both poles appear to have an equivalent temperature of approximately $53 \mathrm{~K}$. Unlike the gas giant planets, with their equatorial minima in tropospheric temperature, and more like Neptune (Fletcher et al. 2014), Uranus' equatorial troposphere is warmer than at mid-latitudes, with a temperature of 53$54 \mathrm{~K}$ at 150 mbar. In the lower stratosphere, at the upper limit of our altitude sensitivity, the warmest temperatures are found near the equator (56-58 $\mathrm{K}$ at 30 
mbar). The 1-bar temperatures approach $80 \mathrm{~K}$, consistent with the more sensitive spectral modeling of Orton et al. (2014b). These Voyager-era temperature profiles will be crucial in simulating synthetic images for comparison with the ground-based imaging.

The distribution of para- $\mathrm{H}_{2}$ also affects the $350-600 \mathrm{~cm}^{-1}$ CIA continuum by increasing the relative strengths of the $S(0)\left(354 \mathrm{~cm}^{-1}\right)$ and $S(1)\left(587 \mathrm{~cm}^{-1}\right)$ absorptions. The para- $\mathrm{H}_{2}$ distribution shown in Fig. $6 \mathrm{~b}$ appears rather different from that derived by Conrath et al. (1998) - we find a tropospheric asymmetry, with higher values of $f_{p}$ in the winter hemisphere (poleward of $40^{\circ} \mathrm{N}$ ) than the summer hemisphere (poleward of $30^{\circ} \mathrm{S}$ ), suggesting an inter-hemispheric transport from the summer to the winter hemisphere. The coolest tropospheric temperatures coincide with minima in the departure of $f_{p}$ from its local equilibrium value, the disequilibrium $\mathrm{f}_{\mathrm{p}}$ (Fig, 6d), consistent with mid-latitude uplift in both hemispheres, and the equator shows a broad maximum in disequilibrium $\mathrm{f}_{\mathrm{p}}$ consistent with atmospheric subsidence. Conrath et al. (1998) showed a similar asymmetry with a higher $\mathrm{f}_{\mathrm{p}}$ in the winter hemisphere, but did not resolve the small-scale structure presented here. We find that our retrieved $f_{p}$ is less than that expected from thermochemical equilibrium throughout the troposphere (with the exception of the equatorial tropopause where $f_{p}>f_{p}$,eqm, suggesting super-equilibrium conditions), and that these sub-equilibrium conditions are strongest $\left(f_{\mathrm{p}}-\mathrm{f}_{\mathrm{p}, \mathrm{eqm}}<-0.05\right.$ at $\left.500 \mathrm{mbar}\right)$ at mid-latitudes (Fig. 6d) in the location of the coolest temperatures (Fig. 6a). This is consistent with upwelling at mid-latitudes, as first determined by Flasar et al. (1987). However, our retrieved meridional $f_{p}$ variation differs from that of Conrath et al. (1998), who found strong super-equilibrium conditions $\left(f_{p}-f_{p, e q m}>0.06\right)$ throughout the winter hemisphere and sub-equilibrium conditions in the summer hemisphere. This difference is related back to the temperature field, which is used to calculate $f_{p, e q m}$, and which shows smaller spatial contrasts in the inversions presented by Conrath et al. (1998). Finally, we note that simultaneous temperature and para- $\mathrm{H}_{2}$ inversions are extremely sensitive to the choice of smoothing constraint, and whilst every effort has been made to limit our sensitivity to the prior, we cannot guarantee that the differences between our new analysis and that of Conrath et al. do not simply arise as a result of differences in retrieval methodology.

Finally, a sensitive test of the appropriateness of the $T(p)$ and $f_{p}(p)$ profiles is in their ability to reproduce center-to-limb structure. Although we were unable to identify specific center-to-limb scans in the Uranus dataset, we coadded spectra in the $50-80^{\circ} \mathrm{N}$ latitude range in emission angle bins. Fig. 7a shows the spectral radiance as a function of wavenumber and emission angle, together with an interpolated representation in Fig. $7 b$. The mean $T(p)$ and $f_{p}(p)$ profiles at this latitude were used to simulate the expected radiance for the same range of emission angles, shown in Fig. 7c. As we can see, the derived profiles reproduce the measurements in a qualitative sense, providing confidence that our spectral inversion has provided results consistent with the full range of constraint offered by the Voyager IRIS data. 


\subsection{Comparison with Voyager IRIS results after one season}

\subsubsection{Generation of Synthetic Images}

To ensure an accurate comparison of Uranian conditions at summer solstice in 1986 with those measured closer to the northern spring equinox, we used the zonal mean temperature and $f_{p}$ distributions to generate synthetic images with the same observing geometry and plate scale as the data described in Section 2. For each image and instrument, we determined the latitude, longitude and emission angle associated with each pixel, using the appropriate plate scale and orientation of Uranus. The Voyager-era atmosphere was used to forward-model radiances in each of the five filters (convolving the filter function with the high-resolution kdistributions). Stratospheric temperatures were assumed to be spatially uniform, following the prior determined by Spitzer observations (Orton et al. 2014b). The resulting synthetic images were convolved with idealized point spread functions representing the stellar images acquired with each Uranus image. These synthetic stellar images were based on a convolution of a diffraction pattern and a seeing pattern, using a full-width half maximum value that best fit the stellar images. Finally, central meridian radiance scans for each filter were extracted in an identical manner with the synthetic images and the real data, in an attempt to look for thermal changes in the intervening two decades. A comparison of these central meridian radiances is shown in Fig. 8.

\subsubsection{Discussion}

Figure 8 reveals a good agreement between our observations and Voyager-IRISbased predictions of meridional variations of radiance along the central meridian of Uranus. This agreement is within the nominal uncertainties associated with nearly all the measurements at any of the wavelengths. We note that the uncertainties shown by the error bars in Fig. 8 represent only the error of the mean over pixels that cover a $\sim 0.25$-arcsec-wide band that is centered at Uranus' central meridian. They do not account for uncertainties in replicating the true point-spread function based on our simulations of the point-spread functions of a nearby standard star. Indeed, the most prominent exceptions to the agreement between the observations and the Voyager IRIS-based simulations are associated with either (i) substantial latitudinal gradients near the near-equatorial peak emission or at the planetary limbs or (ii) in the 24.5- $\mu \mathrm{m}$ image, which has the lowest diffraction-limited spatial resolution. This agreement further validates our decision not to attempt an independent retrieval. Had we done so and used the Voyager temperature and $f_{\text {para }}$ values as a prior guess, we would have found little significant deviation from it.

This agreement that we find extends to the hemispheric asymmetry in temperature that was noted by Conrath et al. (1998) in their analysis of Voyager IRIS observations and which our re-analysis verifies (see section 3.1). They noted that the hemispheric temperature difference involved is quite small, on the order of just $\sim 1 \mathrm{~K}$, a result of the very long radiative time constant at these pressures in Uranus' atmosphere relative to its 84-year orbital period, estimated by Conrath et al. (1990, 
1998) as 130 years at a pressure of 300 mbar or 260 years at a pressure of 100 mbar. Such a small value of the temperature variation is consistent with recent global-climate-models (GCMs) of Uranus by Sussman (2011) and Sussman et al. (2012). In the spectral range of our observations, temperature differences of $1 \mathrm{~K}$ would generate radiance differences of $20-30 \%$ and thus should be easily detectable. For those images taken close to the late-2007 equinox when the effects of different emission angles is minimal (18.7 $\mu \mathrm{m}, 2006$ and $24.5 \mu \mathrm{m}$, in 2008), the north-south asymmetry with a colder northern hemisphere is qualitatively quite evident.

However, as noted originally by Conrath et al. (1990), if this part of the atmosphere is in radiative equilibrium, we would have expected around this epoch ("northern spring equinox") that temperatures in the upper troposphere would be more or less symmetric around the equator (see their Fig. 5a), presuming that the local value of the net upwelling radiance, which they characterize by an equilibrium temperature $\left(\mathrm{T}_{\mathrm{E}}\right)$, is independent of latitude. Conrath et al. (1998), commenting on the discrepancy between the observed hemispherical asymmetry and symmetry expected from considering purely radiative equilibrium, suggested that the influence of longer time constants at deep levels would alter the phasing of the temperature change with insolation. However, we do not detect a different phasing in the observations we report here - we fail to detect any significant change from the distribution of temperatures at the 1986 Voyager epoch of mid-northern winter whatsoever. It does not make sense that this part of the atmosphere would only be influenced by convective energy transport, as it includes a portion of the temperature structure that is far from adiabatic. In fact, it is close to isothermal in the pressure range to which we are sensitive (see Fig. 2). Sussman's full GCM models, which assume that no internal heat is emitted from the deep atmosphere and incorporate both convective and radiative transport, show a minimum temperature near this epoch ("southern autumn equinox") near 20-30S with an extremely shallow increase toward northern latitudes (less than a $2 \mathrm{~K}$ increase from the minimum to the north pole), which is inconsistent with the observed temperature and para- $\mathrm{H}_{2}$ conditions. In theory, the observed lower radiances could be the result of additional opacity from particles in regions of upwelling winds rather than colder temperatures, but the fact that the IRIS instrument detected these at wavelengths as long as $200 \mu \mathrm{m}$ would require the particles to be unrealistically large. An alternative explanation is the possibility that the net upwelling radiance (also expressed as $\mathrm{T}_{\mathrm{E}}$ ) is not meridionally constant, but is lower at northern midlatitudes and is time invariant. A detailed quantitative determination of the meridional variability that is required to match the observed temperature and $f_{p}$ profiles is beyond the scope of this article, but the meridional variations in $\mathrm{T}_{\mathrm{E}}$ would need to be on the same order of the detected 1-3K temperature changes, assuming they are approximately time invariant. This requires that even the small temperature variability predicted by the radiative models (Conrath et al. 1998) and the GCMs of Sussman (2011) and Sussman et al. (2012), all of which have the wrong phase, are overestimated. If radiative equilibrium dominates control of temperatures at these pressures, even longer radiative time constants are required 
than characterized by these models to produce no detectable difference from the IRIS results above our noise level. Conrath et al. (1998) characterize the amplitude of seasonal changes as reduced by $\sqrt{\left(1+r^{2}\right)}$ compared with the $10 \mathrm{~K}$ difference between winter and summer hemispheres for instantaneous radiative equilibrium, where $r=2 \pi t_{R} / t_{0}\left(t_{R}\right.$ is the radiative time constant and $t_{0}$ is the orbital period). The most stringent comparison of our data and the Voyager simulation considers the highest SNR observations from the Keck LWR instrument at $22.0 \mu \mathrm{m}$ (Fig. 8). For these data, the maximum radiance difference is equivalent to a brightness temperature difference of $\sim 0.4 \mathrm{~K}$. This would imply a value for $t_{\mathrm{R}}$ that must be no less than $\sim 330$ years, significantly higher than the 130-year estimate of Conrath et al. $(1990,1998)$. We also note that neither the increase of dynamical activity noted recently in Uranus (e.g. Sromovsky et al. 2012; de Pater et al. 2015) nor longer-term changes noted by Irwin et al. (2012) can be attributed to large-scale changes of temperature field, at least at the resolution of our measurements.

The ultimate test of the meridional variability of $\mathrm{T}_{\mathrm{E}}$ could be addressed by a spacecraft that could determine not only the full bolometric output of the atmosphere as a function of latitude - including the long wavelengths at the peak of the Planck function that the Voyager-2 IRIS experiment did not sample - but also the meridional variability of the Bond albedo - including an estimate of differences in the phase functions characteristic of each latitude. Prior to such a detailed investigation, we can make whatever estimates are possible from the Voyager imaging data themselves, which must include an evaluation of meridional variability in the phase function. We can also determine whether the meridional variability of zonal-mean temperatures remains constant as the season on Uranus approaches northern summer solstice in $2029\left(\mathrm{~L}_{\mathrm{s}}=90^{\circ}\right)$. Similar spatially resolved measurements of thermal emission with better signal-to-noise ratios are recommended. Spatially resolved imaging or spectroscopy using the MIRI instrument on NASA's James Webb Space Telescope (JWST) covering the 5-28 $\mu \mathrm{m}$ range is one such approach, although the diffraction-limited spatial resolution of the 6.5-meter primary mirror of JWST (on the order of 0.83 arcsec at a wavelength of 20 $\mu \mathrm{m})$ will be slightly worse than on the telescopes making the observations reported in this paper (on the order of 0.68 arcsec at $20 \mu \mathrm{m}$ ). With current telescopes and existing instrumentation, we would also recommend observations centered at 17.8 $\mu \mathrm{m}$, which sense as high into the radiatively controlled stratosphere as at $24.5 \mu \mathrm{m}$ (Fig. 2) but would have better spatial resolution. Finally, the construction of 30meter-class telescopes with arrayed mid-infrared detectors hold the promise of 20$\mu \mathrm{m}$ diffraction-limited spatial resolution as good as 0.2 arcsec and the prospect of determining temperatures at a spatial resolution exceeding that of the Voyager IRIS experiment.

\section{Conclusions}

Measurements of spatially resolved thermal emission from the disk of Uranus between 2003 and 2011 are observationally consistent with the results of the Voyager IRIS experiment in 1986. Both the IRIS data and our ground-based results 
are consistent with (i) mid-latitude upwelling producing cool temperature and para$\mathrm{H}_{2}$ minima, (ii) a hemispheric asymmetry in both temperature (cooler northern mid-latitudes) and $f_{p}$ (higher in the northern hemisphere), for which we see no evidence of variability from winter solstice to spring equinox. This invariance is at odds with the predictions of radiative-convective-dynamical models and full global climate models that predict some change in the one season separating the two groups of measurements. One solution is to perturb the assumption of these models regarding the local net upwelling radiance (effective temperature) of the planet by allowing it to vary with latitude while requiring radiatively modulated temperatures to be even smaller than predicted from current models, e.g. by invoking an even longer radiative time constant. Both the invariance of temperatures with time and the variability of effective temperature with latitude can be tested by several types of future observations. Clearly an updated radiative-dynamical model that pieces together the evidence we now have from two seasons is sorely needed.

\section{Acknowledgements.}

Support for this work was provided by NASA from its Planetary Astronomy program through an award issued by the Jet Propulsion Laboratory, California Institute of Technology. L. Fletcher acknowledges support from the Royal Society Research Fellowship. We gratefully acknowledge the work of Meri Marutyan, who assisted in the reduction of the 2008 Subaru COMICS data during the course of her Student Independent Research Internship at JPL, while a student at the Glendale Community College, together with the help of M. Line and P. Yanamandra Fisher to expedite other observations during that observing run. J. Sinclair provided helpful comments prior to submission. Some of the data presented herein were obtained in program 2003A-C14LSN at the W.M. Keck Observatory, which is operated as a scientific partnership among the California Institute of Technology, the University of California and the National Aeronautics and Space Administration. The Observatory was made possible by the generous financial support of the W.M. Keck Foundation. The authors wish to recognize and acknowledge the very significant cultural role and reverence that the summit of Mauna Kea has always had within the indigenous Hawaiian community. We are most fortunate to have the opportunity to conduct observations from this mountain. These results are also based, in part, on data acquired with the VISIR instrument on the ESO Telescopes at the La Silla Paranal Observatory under programme IDs 077C-0571(A) and 083.C-0162(A). These results are also based, in part, on data from programs S08B-031 and S11B-024 at the Subaru Telescope, which is operated by the National Astronomical Observatory of Japan.

(C) 2015. All rights reserved. 


\section{Figures}

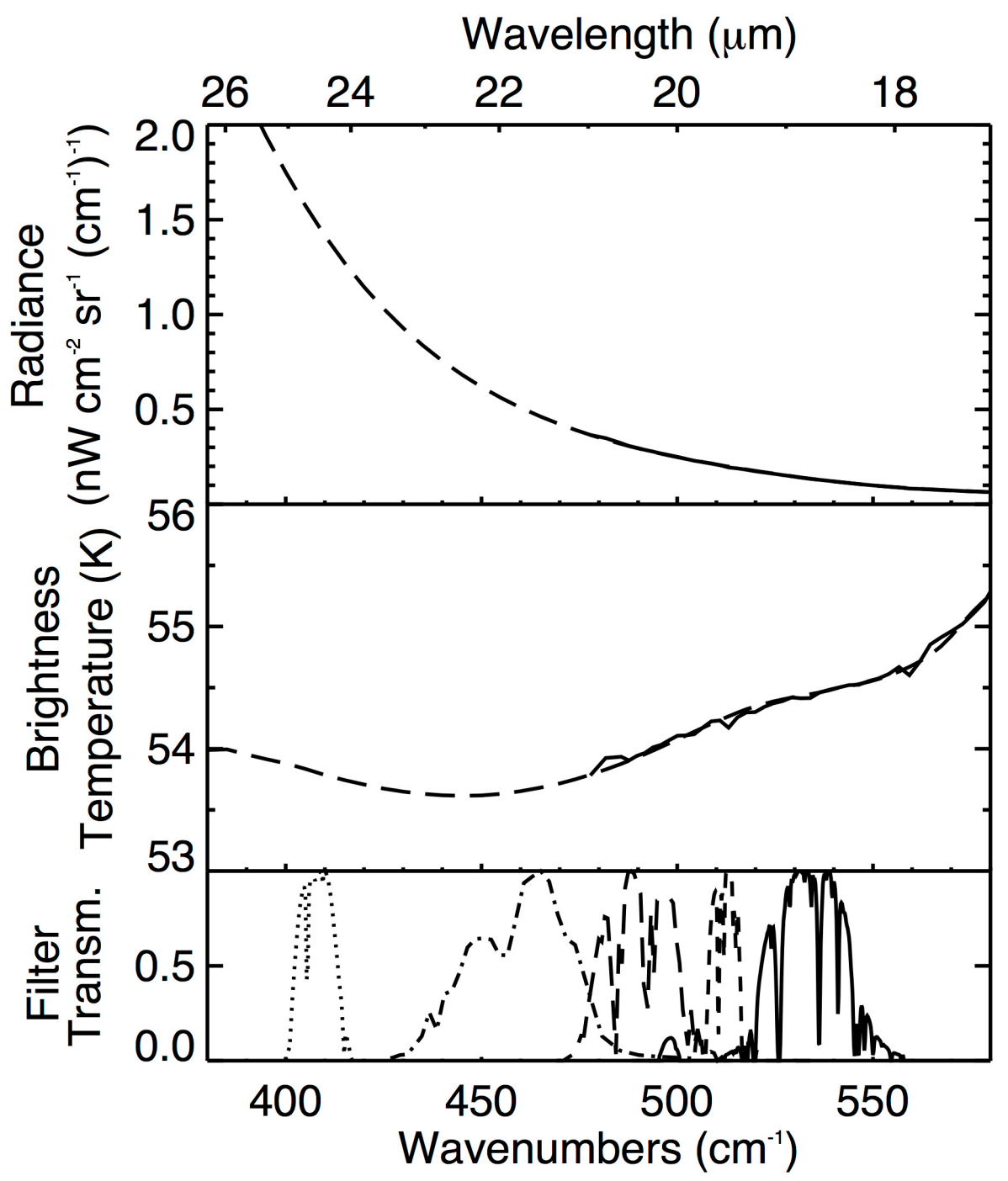

Figure 1. Normalized transmission function of filters relative to the radiance and brightness-temperature spectrum of Uranus. The transmission functions include consideration of telluric absorption, assuming a precipitable water vapor depth of 1 $\mathrm{mm}$. From right to left, the filter functions represent the filters denoted as $18.7 \mu \mathrm{m}$ (solid line), $19.6 \mu \mathrm{m}$ (short-dashed line), $20.3 \mu \mathrm{m}$ (long dashed line), $22.0 \mu \mathrm{m}$ (dashdotted line) and $24.5 \mu \mathrm{m}$ (dotted line). Table 1 summarizes the circumstances of the observations with each filter. The solid line in the radiance and brightnesstemperature plots is the disk-averaged spectrum of Uranus from the long-low mode of Spitzer IRS observations (Orton et al. 2014b). The dashed line is the extension of that spectrum to longer wavelengths using a model that fits the IRS data best. This model assumes a para- $\mathrm{H}_{2}$ fraction that is at equilibrium at the local temperature. 


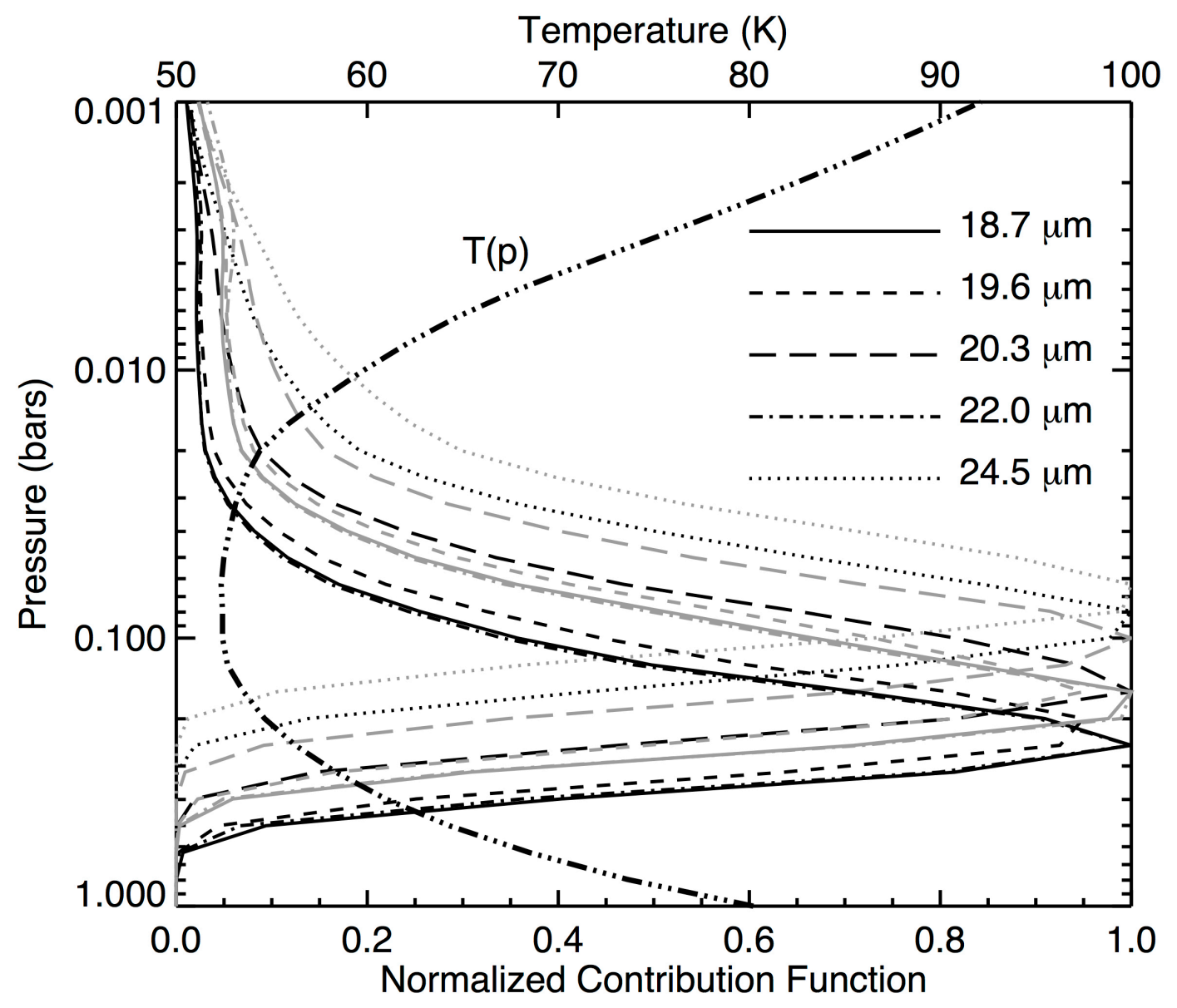

Figure 2. Vertical contribution functions corresponding to the filters shown in Figure 1. Black curves are for emission observed at the nadir, and gray curves are for emission observed at an emission angle of $48^{\circ}$, the highest angle that we estimate that observations at the longest wavelength $(24.5 \mu \mathrm{m})$ detects the planet near the limb without significant contributions from dark space past the limb. The line styles are the same as used in Figure 1. The triple-dotted-dashed curve represents the assumed temperature profile, taken from Orton et al. (2014b). 
OBSERVED

SIMULATED

2006 Sep 2

$18.7 \mu \mathrm{m}$

2006 Sep 3

$18.7 \mu \mathrm{m}$

2009 Aug 27

$19.6 \mu \mathrm{m}$

2011 Aug 27

$20.5 \mu \mathrm{m}$

2003 Jul 27

$22.0 \mu \mathrm{m}$

thens
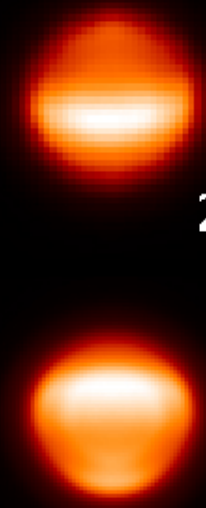
Figure 3. (Previous page) Reduced and simulated images for the wavelengths and epochs described in Table 1 and the text. Reduced images are shown in the lefthand column. To the right of each one is an image of a star that was taken on the same night as a means to display the resolution. Images of the star have been stretched to show details of the combined diffraction and seeing effects. The images of Uranus have been rotated so that north is oriented to the top and are shown without any stretching. These images have had outlying pixels with a standard deviation $>5$ compared with surrounding pixels removed and replaced with the neighborhood average. Otherwise, no deconvolution, optimal filtering, stretching or any other enhancement has been applied to them. For clarity of display, some negative values of the background noise have been suppressed. (No observation of a comparison star was made on 2009 Aug. 27 during VLT service observations using VISIR.) Simulations of the images based on Voyager-2 IRIS derivations of temperature and para- $\mathrm{H}_{2}$ fraction profiles vs. latitude are shown in the next column to the right. Noiseless simulations of the observed stars are shown in the right-hand column. The maximum variation of radiances across the disk is equivalent to $\sim 3 \mathrm{~K}$ of brightness temperature. Radiance and equivalent brightness-temperature scales for zonal means near the central meridians of each of these images are given in Figure 8. 
(a) Emission Angles

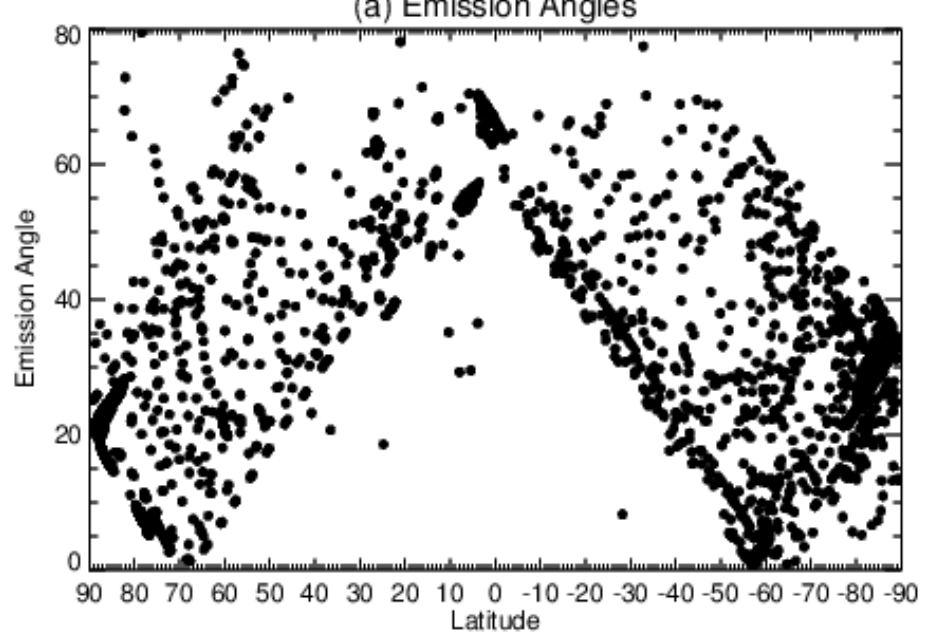

(b) Target Body Semi-Diameter

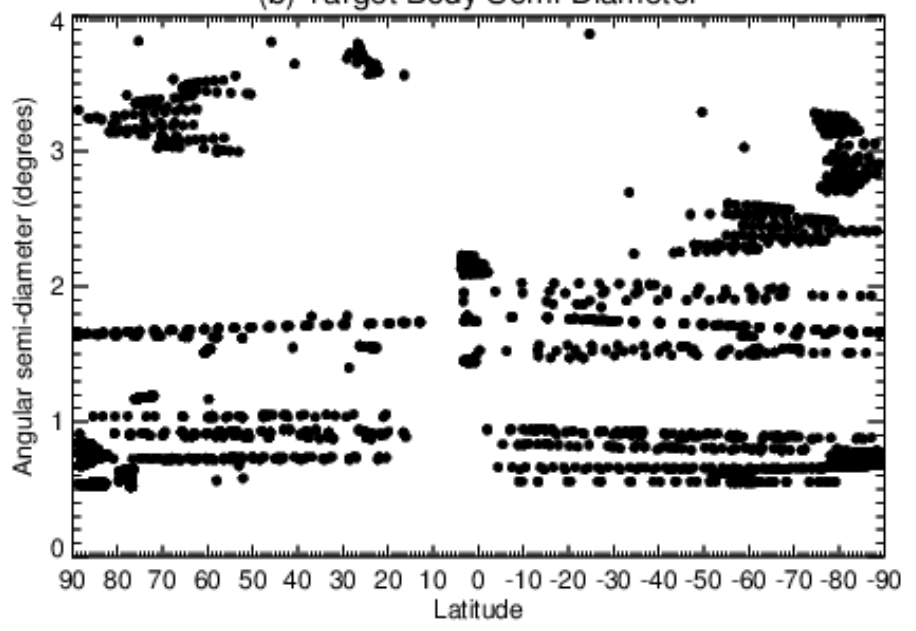

Figure 4. Geometric information for Voyager-2/IRIS data extracted from the Planetary Data System. Spectra were broadly classed as 'closest approach' and 'outbound'. The approach to binning of these data to retrieve zonal-mean temperature and $f_{\text {para }}$ values is described in Section 3.1. 


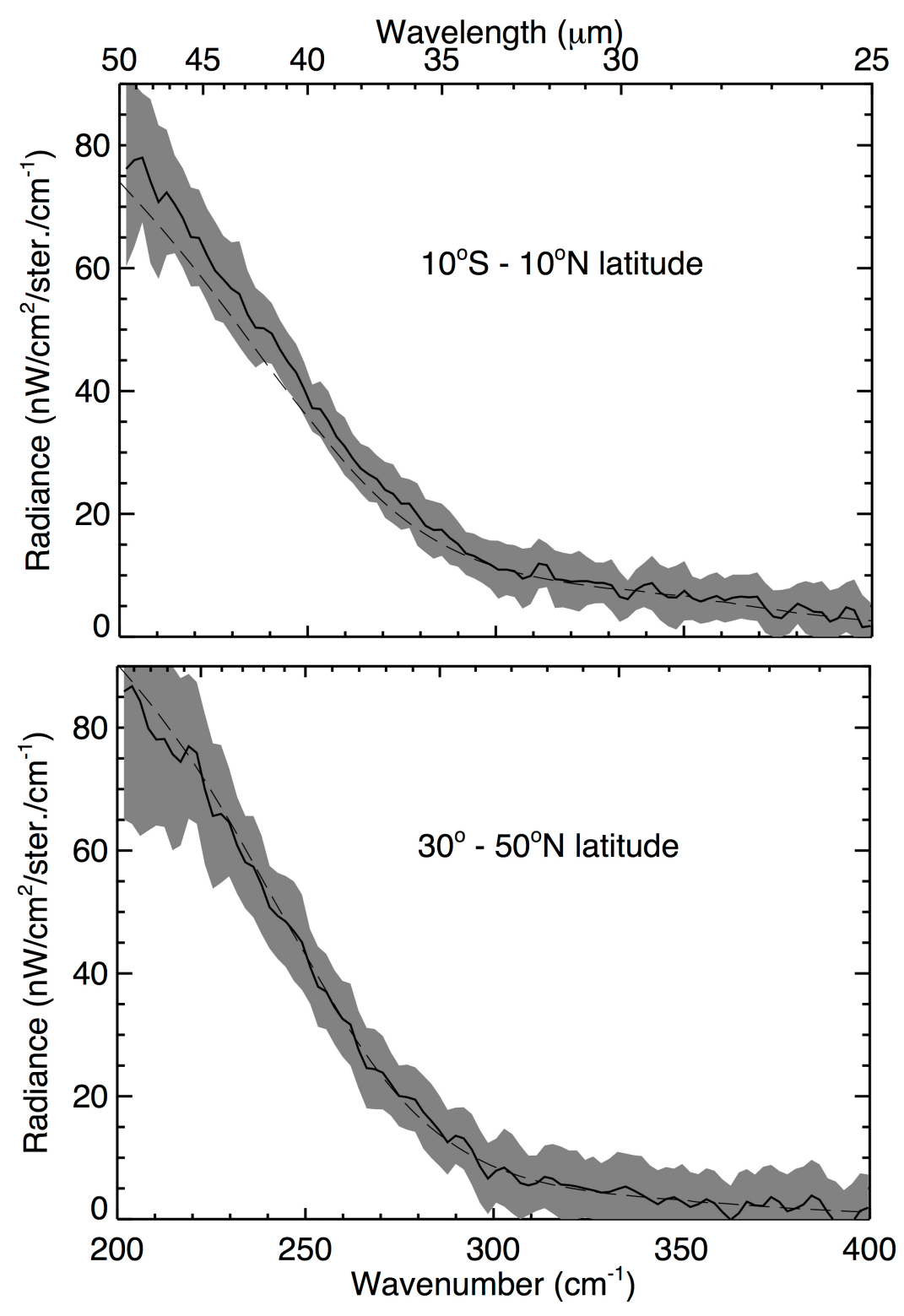

Figure 5. Examples of Voyager-2 IRIS spectra of Uranus. The top panel shows the mean (solid curve) and standard deviation limits (shaded area) of 27 spectra from one of the warmest regions near the equator observed at an average emission angle of $59^{\circ}$; the bottom panel shows the mean and standard deviation of 24 spectra from one of the coolest regions at northern mid-latitudes observed at an average emission angle of $34^{\circ}$. The model spectra corresponding to these regions are shown by the dashed curves. Radiances from the warmest regions (top panel) are lower than those from the coolest regions (bottom panel) as a result of the high emission angles from which they were observed. 
(a) Zonal Mean Temperature [K]

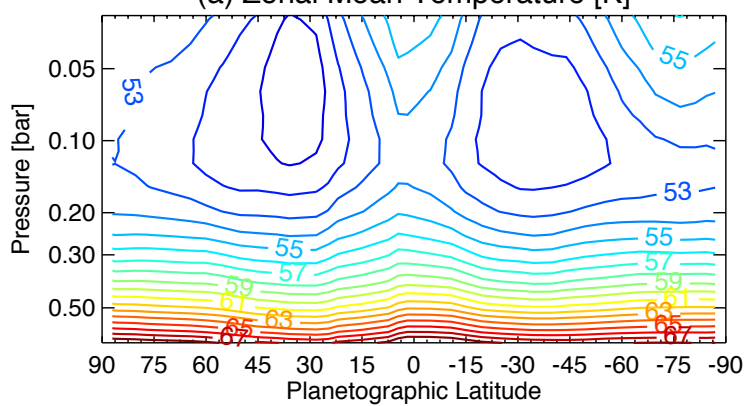

(b) Lapse Rate $[\mathrm{K} / \mathrm{km}]$

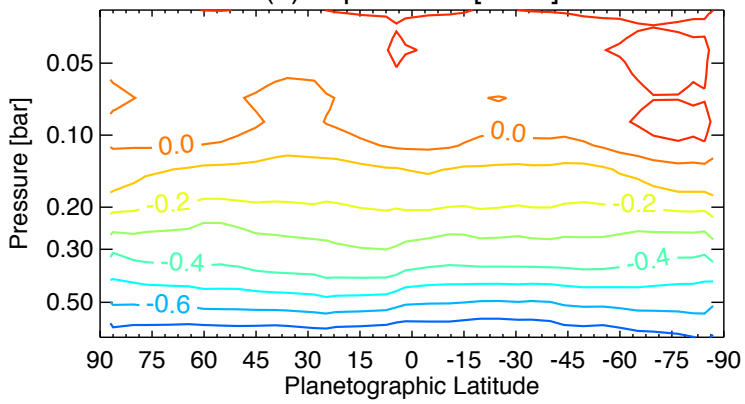

(c) Para- $\mathrm{H}_{2}$ Fraction

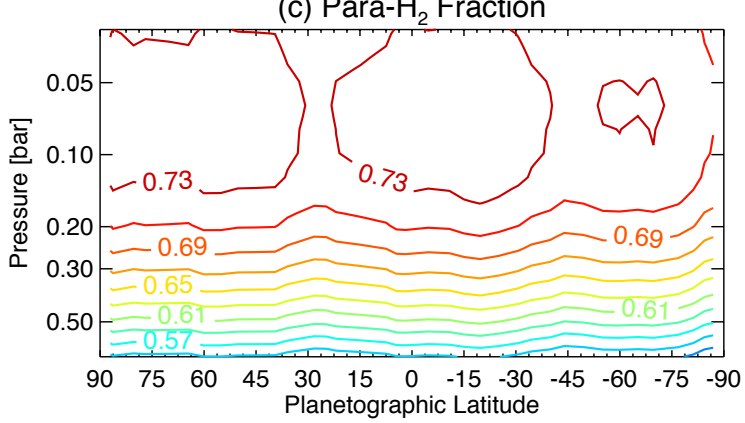

(d) Para- $\mathrm{H}_{2}$ Disequilibrium

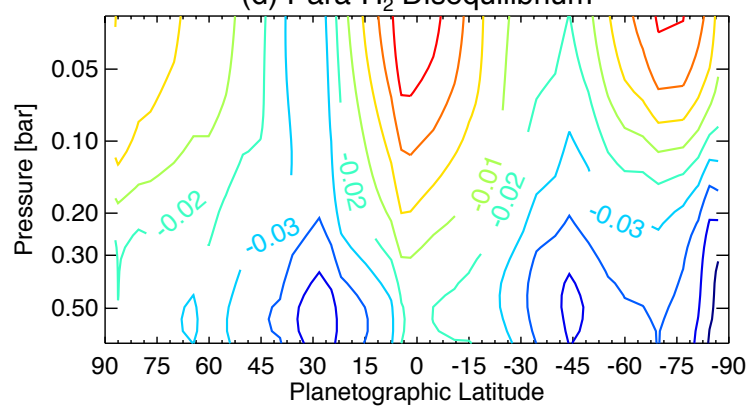

Figure 6. Cross-sections of zonal mean temperatures and para- $\mathrm{H}_{2}$ distributions as derived from Voyager/IRIS spectroscopy in 1986. Panel (a) shows zonal mean temperatures revealing the equatorial maximum and mid-latitude cold regions; and panel (b) shows the lapse rate $(\mathrm{dT} / \mathrm{dz})$ derived from this temperature field, the zero-line indicating the location of the tropopause. Panel (c) shows the zonal mean para- $\mathrm{H}_{2}$ fraction, and panel (d) shows the deviation of the para- $\mathrm{H}_{2}$ fraction from the expectations of thermochemical equilibrium (negative values implying $f_{p}$ is less than $f_{p \text {,eqm }}$ ). Uncertainties on temperatures are smaller than $1 \mathrm{~K}$ and uncertainties on $\mathrm{f}_{\mathrm{p}}$ are smaller than 0.02 . 
(a) Centre-to-Limb [Raw Data]

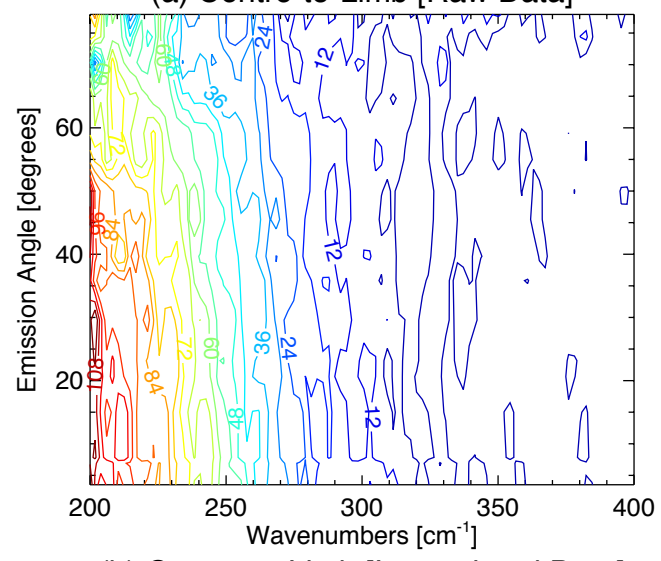

(b) Centre-to-Limb [Interpolated Data]

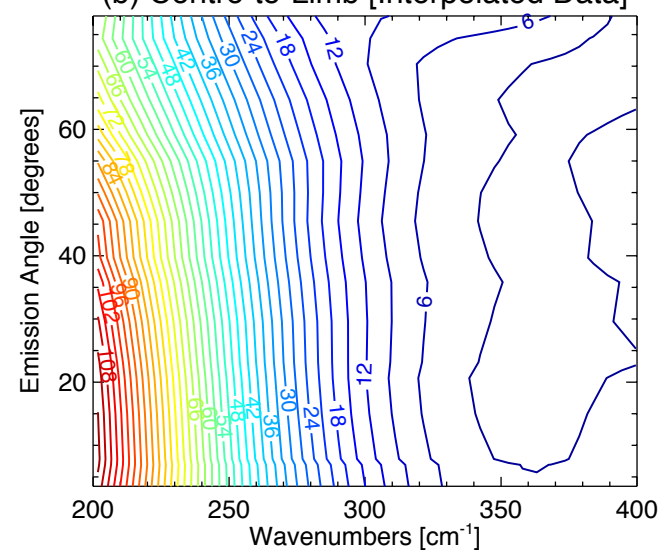

(c) Synthetic Spectra

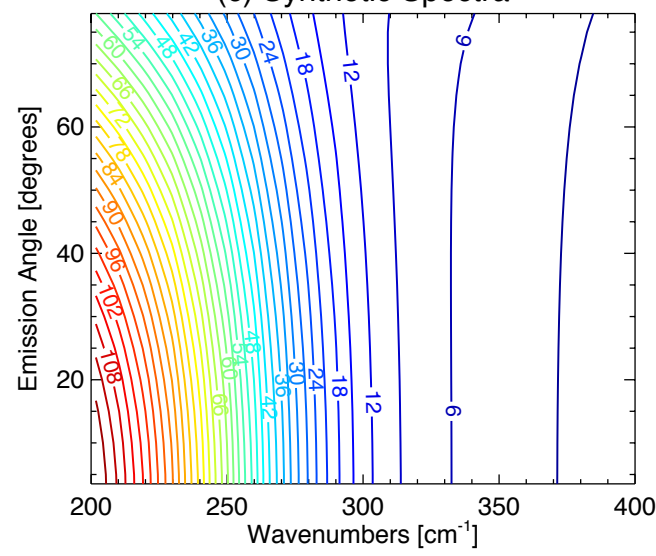

Figure 7. Comparison of center-to-limb measurements with the model and with Voyager2 IRIS data. The top panel shows the raw data, averaged between $50-80^{\circ} \mathrm{N}$, over a range of emission angles. This is smoothed to create the center panel, to facilitate comparison with the synthetic radiances in the bottom panel. Contour units are $\mathrm{nW} / \mathrm{cm}^{2} / \mathrm{sr} / \mathrm{cm}^{-1}$. 
Planetographic Latitude

$60^{\circ} \mathrm{S} 30^{\circ} \mathrm{S} \quad 0^{\circ} 30^{\circ} \mathrm{N} 60^{\circ} \mathrm{N}$
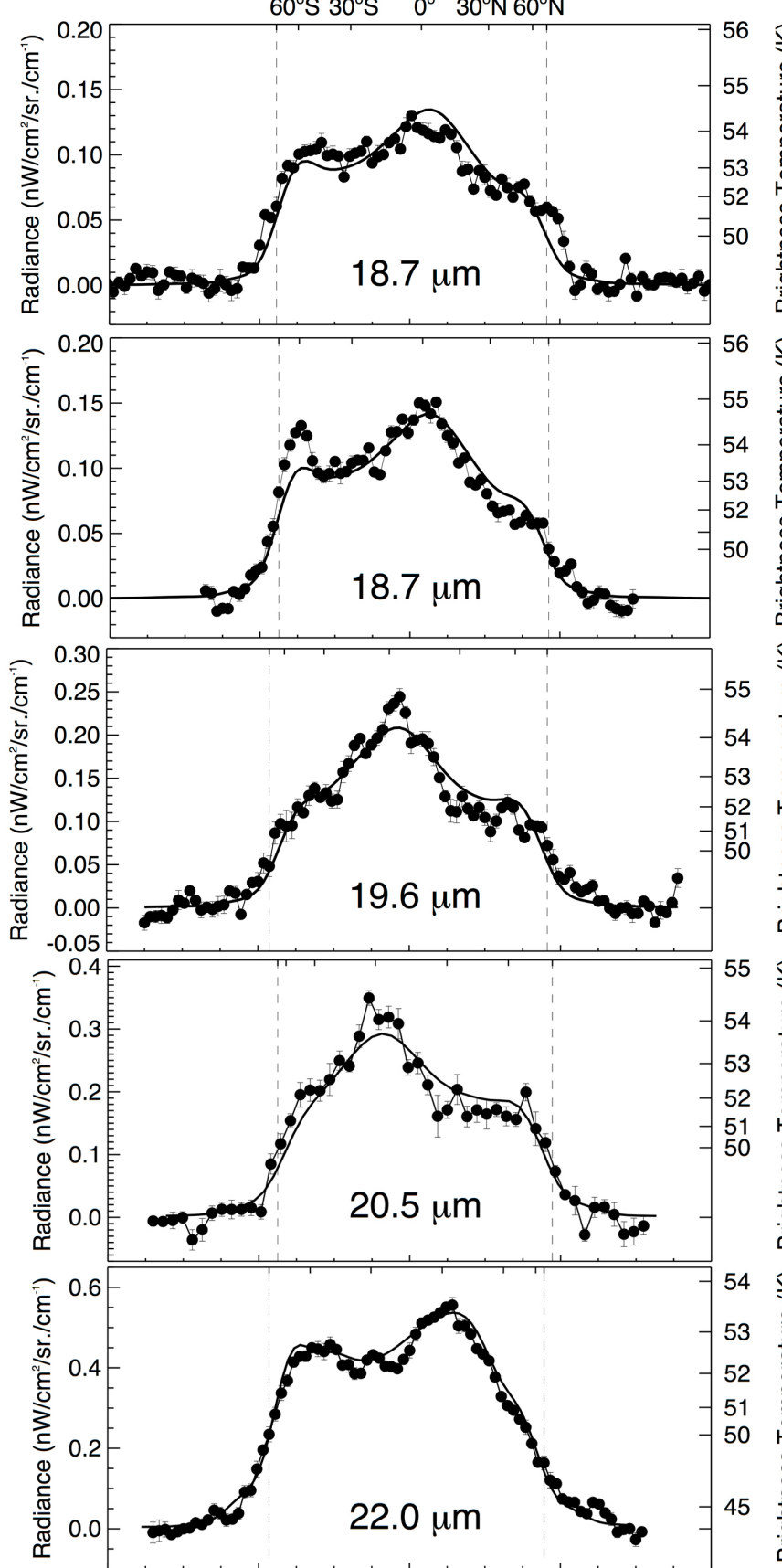

$54 \underline{\underline{s}}$

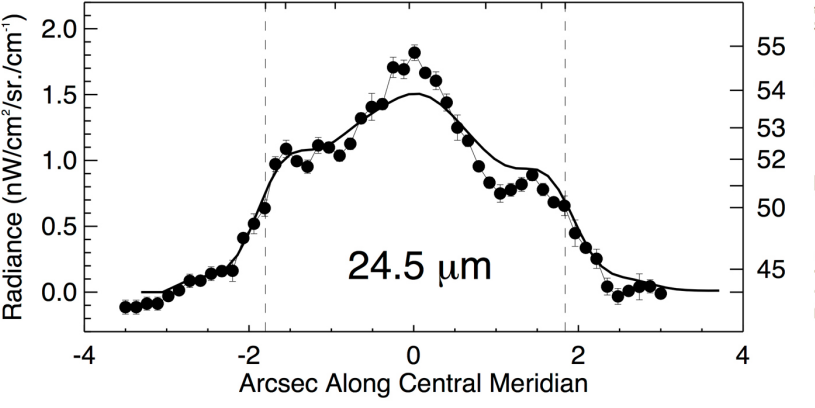

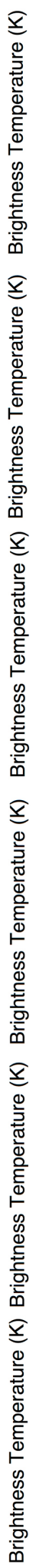


Figure 8 (Previous page) Comparison between the synthetic radiances along the central meridian of Uranus with the measurements, identified by central wavelength in the same order as in Fig. 3. Measurements are marked by thin line, filled circles and with associated uncertainties. The measurement uncertainties are derived from the error of the mean over pixels covering $\sim 0.25$ arcsec, centered on the planetary central meridian. The thicker line represents the predicted central-meridian radiances. The location of corresponding latitude positions is given by the upper abscissa, as labeled on the topmost panel. Note that the latitude tics are different for each wavelength because they were observed at different epochs. The vertical dashed lines schematically represent the north and south limbs of the planet. Lacking a trustworthy independent calibration, the radiance of the measurements has been scaled to the radiance derived from the VoyagerIRIS-based simulation. Equivalent brightness temperatures for the range of radiances on the disk are given on the right-hand ordinate scale. 


\section{References}

Archinal, B. A., A'Hearn, M. F., Bowell, E., Conrad, A., Consolmagno, G. J., Courtin, R., Fukushima, T., Hestroffer, D., Hilton, J. L., Krasinsky, G. A., Neumann, G., Oberst, J., Seidelmann, P. K., Stooke, P., Tholen, D. J., Thomas, P. C., Williams, I. P., 2011. Report of the IAU Working Group on Cartographic Coordinates and Rotational Elements: 2009. Celestial Mechanics and Dynamical Astronomy 109, 101-135.

Borysow, A., Frommhold, L., 1986. Theoretical collision-induced rototranslational absorption spectra for the outer planets: H2-CH4 pairs. Astrophys. J. 304, 849-865.

Borysow, J., Frommhold, L., Birnbaum, G., 1988. Collison-induced rototranslational absorption spectra of $\mathrm{H}_{2}$-He pairs at temperatures from 40 to $3000 \mathrm{~K}$. Astrophys. J. 326, 509-515.

Conrath, B., Hanel, R., Gautier, D., Marten, A., Lindal, G. 1987. The helium abundance of Uranus from Voyager measurements. J. Geophys. Res. 92, 15003-15010.

Conrath, B. J., Gierasch, P. J., Leroy, S. S. 1990. Temperature and circulation in the stratosphere of the outer planets. Icarus 83, 255-281.

Conrath, B. J., Gierasch, P. J., Ustinov, E. A. 1998. Thermal structure and para hydrogen fraction on the outer planets from Voyager IRIS measurements. Icarus 135, 501-517.

de Pater, I., Sromovsky, L. A. Fry, P. M., Hammel, H. B., Baranec, C., Sayanagi, K. M. 2015. Record-breaking storm activity on Uranus in 2014. Icarus 252, 121-128.

Flasar, F. M., Conrath, B. J., Gierasch, P. J. Pirraglia, J. A. 1987. Voyager Infrared observations of Uranus' atmosphere: Thermal structure and dynamics. J. Geophys. Res. 92, 15011-15018.

Fletcher, L. M., Orton, G. S., Yanamandra-Fisher, P. Fisher, B. M., Parish, P. D., Irwin, P. G. J. 2009. Retrievals of atmospheric variables on the gas giants from ground-based mid-infrared imaging. Icarus 200, 154-175.

Fletcher, L.N., de Pater, I., Orton, G.S., Hammel, H.B., Sitko, M.L., Irwin, P.G.J.. 2014. Neptune at Summer Solstice: Zonal Mean Temperatures from Ground-Based Observations, 2003-2007. Icarus 231, 146-167.

Irwin, P., Teanby, N., de Kok, R., Fletcher, L., Howett, C., Tsang, C., Wilson, C., Calcutt, S., Nixon, C., Parrish, P. 2008. The NEMESIS planetary atmosphere radiative transfer and retrieval tool. J. Quant. Spectrosc. \& Radiative Transf. 109, 1136-1150. 
Irwin, P. G. J., Teanby, N. A. Davis, G. R., Fletcher, L. N., Orton, G. S., Calcutt, S. B, Tice, D. S., Hurley, J. 2012. Further seasonal changes in Uranus' cloud structure observed by Gemini-North and UKIRT. Icarus 218, 47-55.

Jones, B. and Peutter, R. C. 1993. Keck long-wavelength spectrometer. In Jones, B., Puetter, R. C. (Eds.) SPIE 1946, Infrared Detectors and Instrumentation, (Albert M. Fowler, ed.), pp. 610-621.

Karkoschka, E. and Tomasko, M. 2009. The haze and methane distributions on Uranus from HST-STIS spectroscopy. Icarus 202, 287-309.

Karkoschka, E. and Tomasko, M. 2011. The haze and methane distributions on Neptune from HST-STIS spectroscopy. Icarus 211, 780-797.

Karkoschka, E. 2014. Uranus' southern circulation revealed by Voyager-2 images: Asymmetric, unique, unexpected. Bull. Amer. Astron. Soc. 46, 224.

Kataza, H., Okamoto, Y., Takubo, S., Onaka, T., Sako, S., Nakamura, K., Miyata, T., Tamashita, T., 2000. COMICS: the cooled mid-infrared camera and spectrometer for the Subaru Telescope. Proc. SPIE 4008, 1144-1152.

Lagage, P. O., Pel, J. W., Authier, M., Belorgey, J., Claret, A., Doucet, C., Dubreuil, D., Durand, G., Elswijk, E., Girardot, P., Käufl, H. U., Kroes, G., Lortholary, M., Lussignol, Y., Marchesi, M., Pantin, E., Peletier, R., Pirard, J., Pragt, J., Rio, Y., Schoenmaker, T., Siebenmorgen, R., Silber, A., Smette, A., Sterzik, M., Veyssiere, C. 2004. Successful commissioning of VISIR: The mid-Infrared VLT instrument. The Messenger 117, 1216.

Lindal, G. F., Lyons, J. R., Sweetnam, D. N., Eshleman, V. R., Hinson, D. P. 1987. The atmosphere of Uranus - Results of radio occultation measurements with Voyager 2. J. Geophys. Res. 92, 14987-15001.

Orton, G. S., Encrenaz T., Leyrat, C., Pantin, E., Puetter, R. and Friedson, A. J. 2007a. Evidence for strong seasonal and dynamical perturbations of Neptune's temperature field. Astron. \& Astrophys. 473, L3-L8.

Orton, G. S., Gustafsson, M., Burgdorf, M., Meadows, V., 2007b. Revised Ab Initio Models for $\mathrm{H}_{2}-\mathrm{H}_{2}$ collision induced absorption at low temperatures. Icarus 189, 544-549.

Orton, G. S., Fletcher, L. N., Moses, J. I., Mainzer, A., Hines, D., Hammel, H. MartinTorres, J., Burgdorf, M., Merlet, C., Line, M. R. 2014a. Mid-Infrared spectroscopy of Uranus from the Spitzer Infrared Spectrometer: 2. Determination of the mean composition of the upper troposphere and stratosphere. Icarus. 243, 471-493. doi 10.1016/j.icarus.2014.07.010. 
Orton, G. S., Moses, J. K., Fletcher, L. N., Mainzer, A., Hines, D., Hammel, H. MartinTorres, J., Burgdorf, M., Merlet, C., Line, M. R. 2014b. Mid-Infrared spectroscopy of Uranus from the Spitzer Infrared Spectrometer: 1. Determination of the mean temperature of the upper troposphere and stratosphere. Icarus. 243, 494-513. doi 10.1016/j.icarus.2014.07.012.

Rages, K., Pollack, J. B., Tomasko, M. G., Doose, L. R. 1991. Properties of scatterers in the troposphere and lower stratosphere of Uranus based on Voyager imaging data. Icarus 89, 359-376.

Sromovsky, L. A., Fry, P. A., Kim, J. H. 2011. Methane on Uranus: The case for a compact $\mathrm{CH}_{4}$ cloud layer at low latitudes and severe depletion at high latitudes baed on re-analysis of Voyager occultation measurements and STIS spectroscopy. Icarus 215, 292-312.

Sromovsky, L. A., Fry, P. M., Hammel, H. B., de Pater, I., Rages, K. A. 2012. Postequinox dynamics and polar cloud structure on Uranus. Icarus 230, 694-712.

Sussman, M. G. 2011. Modeling seasonal change on Uranus with the EPIC GCM. Ph.D. Thesis. New Mexico State University.

Sussman, M. G, Dowling, T. E., Chanover, N. J., Greathouse, T. K. 2012. Bull. Amer. Astron. Soc. 44, 252-253. 